\title{
A Study of Chinese-English Menu Translation in Light of Skopos Theory Xiao-Qing SUN ${ }^{1} \mathrm{a}^{*}$, Jia-Lu SUN ${ }^{1}$ \\ ${ }^{1}$ Shanghai University of Engineering Science, China \\ acntysxq@163.com
}

Keywords: Menu Translation, Skopos Theory

\begin{abstract}
Based on the analysis of main viewpoints of Skopos Theory, this paper puts forward two principles of Chinese-English menu translation and recommends five specific strategies.
\end{abstract}

\section{Introduction}

With the increasing frequency of China's foreign exchange activities, cultural translation has attracted more and more attention. China's food culture as an important part of Chinese culture, has aroused the concern of the community. China Knowledge Network (CNKI) Chinese journal full-text database search shows that about 2700 copies of the relevant literature were published in last 10 years. It shows that the domestic translation industry has attached great importance to the translation of menus.

\section{Theoretical Basis}

Skopos is derived from Greek, meaning "purpose". It is an important part of the German functional translation school, which is composed of Katharina Reiss and Hans J. Vermeer in the 1970s. In 1984, Fermil and his mentor, the founder of the German functional school Katrina Rice worked together and completed the book "General Translation Theory Foundation". They suggested that the decisive factor in the translation strategy was not the function of the source, but rather the intended function of the translation. In the view of Fermil, translation is the conversion of language and nonverbal symbols in different language types.

From this perspective, the translation strategy of the Chinese cuisine is not focused on the primitive function of Chinese dishes - to provide some information for Chinese diners, but rather to the intended function of foreign language translations - to allow foreign diners to enjoy Chinese food while enjoying. As a cross-cultural act, there is a strong Chinese cultural orientation, which determines that the purpose of translation is to obtain the necessary basic information for foreign diners. Translation emphasizes the reader as the center and the target language culture as the guide.

\section{Principle of Chinese-English Menu Translation}

Based on the extensive investigation of menus, on the basis of summarizing and summarizing the translation problems, the author thinks that Chinese-English menu translation should follow the following principles.

\section{To Achieve the Function-Oriented Menu}

The concept of "function + loyalty" proposed by Nord allows the translator to adjust the necessary functions to achieve the intended function of the translation on the basis of analyzing the original text, including changing the content and stylistic features of the original text. Therefore, the primary purpose of the recipe translation is to achieve its function. The author believes that Chinese dishes also has a cultural transmission and aesthetic functions. In the practice of menu translation, the translator should first ensure that the information function. On this basis, the cultural transmission and aesthetic function should be realized as much as possible. Therefore, the translator should first ensure that diners can understand the dishes of raw materials, cooking methods and other basic information. If possible, describe the shape of the dishes, taste, rhetorical devices, contains allusions 
and aesthetic characteristics of information to attract the attention of the patrons, to stimulate their desire to buy.

\section{To Understand Cultural Differences and Avoid Cultural Conflicts}

Chinese culture in the cultural information contained in the process of language conversion is easy to cause ambiguity. We should consider the differences between Chinese and Western cultures. For the target language readers to understand the more difficult cultural information, the translator must abandon it, only to retain the basic information of the dishes.

\section{Strategies of Chinese-English Menu Translation}

1) main ingredients with juice supplement

Such as Chinese Mushrooms with Pine Nuts

2) cooking way and raw materials

Such as Tossed Black and White Fungus, Sautéed Spicy Beef and Green Peas and Tossed Clear Noodles with Chili Sauce

3) shape and taste with raw materials

Such as Rabbit-Shaped Mantou, Crispy Chicken Crispy Chicken, and Sautéed Sliced Lamb with Pepper and Parsley

4) name and place names with raw materials

Such as Mapo Tofu (Sautéed Tofu in Hot and Spicy Sauce), Cantonese Dim Sum, Spicy Chicken, Sichuan Style and Noodles with Soy Bean Paste, Beijing Style.

5) Chinese Pinyin with annotation

Such as Jiaozi, Baozi, Mantou, Huajuan,Shaomai, Fotiaoqiang ( Steamed Abalone with Shark's Fin and Fish Maw in Broth ), Guotie ( Pan-Fried Dumplings ), Wotou ( Steamed Corn/Black Rice Bun ), Steamed Jiaozi ( Steamed Dumplings ), Youtiao ( Deep-Fried Dough Sticks ), Tangyuan ( Glutinous Rice Balls ) and Zongzi ( Glutinous Rice Wrapped in Bamboo Leaves )

\section{Conclusion}

The above principles and strategies provide a new perspective for Chinese-English menu translation. The author first tries to ensure that the menu function can be achieved, and meanwhile tries to retain its rhetoric and cultural characteristics with the cultural information and aesthetics characteristics, so that more foreign guests can understand and appreciate the Chinese food culture.

\section{References}

[1] Lu Jing, Mao Ping. On English Translation Principles and Methods of Chinese Menus from the Perspective of Skopos Theory [J]. Journal of Huaihai Institute of Technology (Humanities and Social Sciences), 2016 (7): 60-62

[2] Ren Jingsheng. The Problem of Chinese Translation of Chinese Food and Staple food [J]. Chinese translation, 2001 (6): 56-58.

[3] Liu Qingbo. Translation of Chinese Cuisine and the Principle of Translation [J]. Chinese Science and Technology Translation, 2003 (4): 52-54.

[4] Chen Xiaowei. New Practical Translation Course [M]. Beijing: Economic Science Press, 2006.

[5] Beijing Municipal People's Government Foreign Affairs Office. Enjoying Culinary Delights: Beijing: World Knowledge Publishing House, 2012. [M]. Beijing: World Knowledge Publishing House, 2012.

[6] Liu Huan. Festival Cookbook Translation: Imagery Beauty and Pragmatic Equivalent Function of the Combination [J] .Guangdong Institute of Education, 2008 (6): 89- 92. 